University of Nebraska - Lincoln

DigitalCommons@University of Nebraska - Lincoln

\title{
Colossal Dielectric and Electromechanical Responses in Self Assembled Polymeric Nanocomposites
}

\author{
Cheng Huang \\ Pennsylvania State University, University Park, Pennsylvania \\ Q. M. Zhang \\ Pennsylvania State University, University Park, Pennsylvania \\ Jiang Yu Li \\ University of Nebraska - Lincoln \\ Manese Rabeony \\ Rutgers University, Piscataway, New Jersey
}

Follow this and additional works at: https://digitalcommons.unl.edu/engineeringmechanicsfacpub

Part of the Mechanical Engineering Commons

\begin{abstract}
Huang, Cheng; Zhang, Q. M.; Yu Li, Jiang; and Rabeony, Manese, "Colossal Dielectric and Electromechanical Responses in Self Assembled Polymeric Nanocomposites" (2005). Faculty Publications from the Department of Engineering Mechanics. 43.

https://digitalcommons.unl.edu/engineeringmechanicsfacpub/43
\end{abstract}

This Article is brought to you for free and open access by the Mechanical \& Materials Engineering, Department of at DigitalCommons@University of Nebraska - Lincoln. It has been accepted for inclusion in Faculty Publications from the Department of Engineering Mechanics by an authorized administrator of DigitalCommons@University of Nebraska - Lincoln. 


\title{
Colossal dielectric and electromechanical responses in self-assembled polymeric nanocomposites
}

\author{
Cheng Huang and Q. M. Zhang ${ }^{\text {a) }}$ \\ Materials Research Institute and Electrical Engineering Department, The Pennsylvania State University, \\ University Park, Pennsylvania 16802 \\ Jiang Yu Li \\ Department of Engineering Mechanics, University of Nebraska-Lincoln, Lincoln, Nebraska 68558 \\ Manese Rabeony \\ Chemistry Department, Rutgers University, Piscataway, New Jersey 08854
}

(Received 3 June 2005; accepted 30 August 2005; published online 24 October 2005)

\begin{abstract}
An electroactive polymer nanocomposite, in which high dielectric constant copper phthalocyanine oligomer (o-CuPc) nanoparticles are incorporated into the block polyurethane (PU) matrix by the combination of "top down" and "bottom up" approaches, was realized. Such an approach enables the nanocomposite to exhibit colossal dielectric and electromechanical responses with very low volume fraction of the high dielectric constant o-CuPc nanofillers $(\sim 3.5 \%)$ in the composite. In contrast, a simple blend of o-CuPc and PU composite with much higher o-CuPc content $(\sim 16 \%$ of $\mathrm{o}-\mathrm{CuPc}$ ) shows much lower dielectric and electromechanical responses. (C) 2005 American Institute of Physics. [DOI: 10.1063/1.2105997]
\end{abstract}

Electroactive polymers (EAPs) with high dielectric constant and high electromechanical responses are promising for advanced electromechanical and dielectric applications such as actuators, artificial muscles, and high efficiency charge storage capacitors. ${ }^{1-5}$ Self-assembly based on the spontaneous control of materials through noncovalent interactions, e.g., hydrogen bonding, van der Waals forces, and electrostatic forces, provides a powerful method for lowdimensional nanoscopic building blocks to assemble complex architectures with novel electronic, magnetic, and photonic properties. ${ }^{6-8}$ In this letter, we will utilize this approach to fabricate electroactive polymer nanocomposites possessing high electromechanical and dielectric responses.

Recently, an all-organic composite actuator material with a high dielectric constant was realized by incorporating high dielectric constant organic filler, i.e., copper phthalocyanine oligomer (o-CuPc), in a polymer matrix. ${ }^{9}$ This all-organic EAP composite exhibits a strain of $\sim 2 \%$ and an elastic energy density of $0.13 \mathrm{~J} / \mathrm{cm}^{3}$ induced by an electric field of $13 \mathrm{~V} / \mu \mathrm{m}$. In this class of EAPs, the high dielectric constant is achieved by a combination of the high dielectric constant of the polymer matrix, the poly(vinylidene fluoridetrifluoroethylene) based relaxor ferroelectric which possesses a dielectric constant higher than 50 , and the very large polarization response due to the delocalized electrons in the o-CuPc. ${ }^{9-11}$ It is known that phthalocyanine as well as its oligomer has a strong tendency to form stack assemblies and microaggregates due to its planar shape and aromatic nature, ${ }^{12-14}$ so it is hard for polymer dielectric matrix to directly disstack these high dielectric constant microaggregates due to the very weak interactions between them. ${ }^{9,15,16}$ Hence, the preparation of the nanometer-scale building blocks, highly dispersed nanoparticles is necessary before selfassembly. Herein, we demonstrate a polymeric nanocompos-

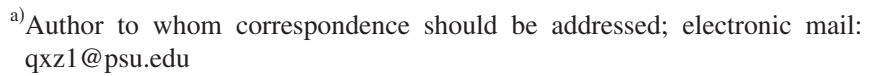

ite in which high dielectric constant o-CuPc nanoparticles were incorporated into the block polyurethane (PU) by the combination of "top-down" and "bottom-up" self-assembly approaches. Even though the PU matrix has a much lower dielectric constant $(<10)$ in comparison with the relaxor ferroelectric polymer matrix, these polymeric nanocomposites still exhibit very large dielectric and electromechanical responses.

The copper-phthalocyanine sheet oligomer (o-CuPc) possessing peripheral carboxylic groups was synthesized by a solution method in high boiling-point solvent. ${ }^{15}$ The topdown approach is based on the spontaneous and strong chemisorptions of poly(acrylic acid), PAA, on the o-CuPc surfaces due to strong electrostatic force and complexation, ${ }^{17,18}$ which constitutes the driving force for the gradual division of the initially large o-CuPc microaggregates into much smaller particles, stabilized by the charged PAA layer, finally leading to highly dispersed colloidal nanoparticles (referred to as PAA-decorated o-CuPc). In this topdown approach, PAA was dissolved in de-ionized water and a proper amount of finely grounded $\mathrm{CuPc}$ oligomer powder (the amount of $\mathrm{CuPc}$ is determined by the PAA/CuPc ratio and in this study, a 50/50 wt $\%$ PAA/CuPc was used, corresponding to $47 / 53 \mathrm{vol} \%$ ) was added into the PAA solution. The solution mixture of CuPc oligomer and PAA was ultrasonically stirred at $90{ }^{\circ} \mathrm{C}$ to dissolve $\mathrm{CuPc}$ oligomer in water via complexation with PAA. Figure 1(a) shows a transmission electron microscopy (TEM) micrograph of the PAAdecorated o- $\mathrm{CuPc}$ particles thus prepared which have the size of about $40 \mathrm{~nm}$.

A bottom-up approach was then utilized to self-assembly of these high dielectric constant PAA-decorated o-CuPc nanoparticles into the PU matrix (with $10 / 90 \mathrm{wt} \%$, $\sim 7.3$ vol \% of PAA-decorated o-CuPc which is sufficient to induce very high dielectric response in the nanocomposite). ${ }^{19}$ The polyether soft segments in block polyurethane can further coat the decorated o-CuPc ${ }^{18,20-22}$ and block polyurethane can act as a template for the self-assembly of these oligomer 

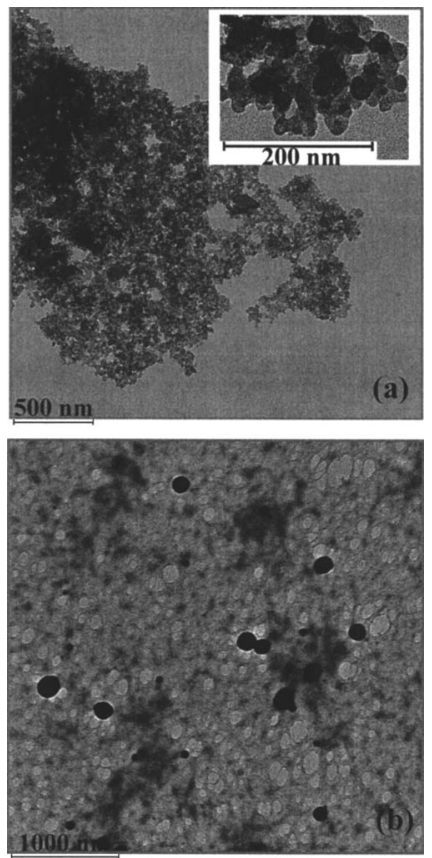

FIG. 1. (a) TEM images of decorated CuPc oligomer nanoparticles. (b) TEM micrograph of the final morphology for the polymeric exchangecoupled nanocomposite film. [Inclusions are uniformly distributed in the matrix (not resolved) and during the TEM sample preparation the TEM microtomy causes some larger aggregation of particles (big dark spots) and air bubbles (white holes).]

nanoparticles. ${ }^{19,23}$ In this process, polyurethane, PU, was dissolved in N,N-dimethylformide (DMF). The mixed PU-DMF solution together with PAA-decorated CuPc oligomer aqueous solution with excess DMF (ten times the volume of water) were mechanically stirred, and then the mixture was poured onto a glass slide and dried in air at $80{ }^{\circ} \mathrm{C}$ and then under vacuum for $12 \mathrm{~h}$. Finally, the nanocomposite was annealed at $140{ }^{\circ} \mathrm{C}$ in a vacuum for $12 \mathrm{~h}$ and slowly cooled down to room temperature. Figure 1(b) shows the TEM micrograph of the self-assembled nanocomposites with decorated $\mathrm{CuPc}$ oligomer nanoparticles in the PU matrix. For comparison, the simple blend of PU and undecorated $\mathrm{CuPc}$ powder with $20 / 80 \mathrm{wt} \%(\sim 16 \mathrm{vol} \%)$ was prepared using the same solution cast procedure with DMF as the solvent. Scanning electron microscopy (SEM) micrograph shows o-CuPc microagglomerates $(\sim 1 \mu \mathrm{m}$ size $)$ in the simple blend composite.

In this work transmission electron microscopy (TEM) and SEM were used to characterize the microstructures and morphology of the composites. For the PAA-decorated CuPc oligomer, the specimen was prepared by placing a drop of a solution on carbon film coated copper grid, then dried in air before observation. For the nanocomposite, TEM analysis was performed on 80-nm-thick film microtomed at room temperature. The samples were coated with $\mathrm{Au}(\sim 3 \mathrm{~nm})$ by vacuum sputtering for SEM observation. In order to investigate the bulk morphology of the polymers, the sample was broken in liquid nitrogen and its cross section was analyzed by SEM. The typical sample film thickness for electric characterization was about $50 \mu \mathrm{m}$ and $\mathrm{Au}$ was sputtered on both surfaces of the sample films as electrodes. The dielectric properties of the samples were evaluated by a HP 4284 LCR meter in the frequency range of $20 \mathrm{~Hz}-1 \mathrm{MHz}$ at room temperature. The elastic moduli were determined using a com-
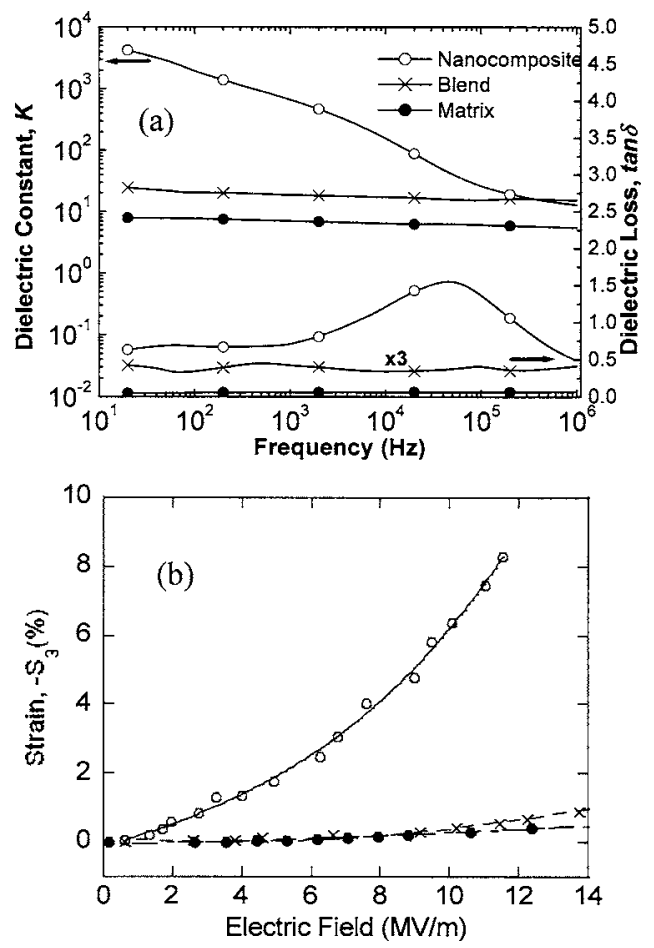

FIG. 2. (a) Dielectric constant and dielectric loss as a function of frequency for the nanocomposite (open circles) measured at room temperature. (b) Electromechanical strain amplitude as a function of the applied-field amplitude for the nanocomposite (open circles), and the applied-field frequency is $1 \mathrm{~Hz}$. Data points are shown and solid curves are a guide to the eye. For comparison, the data from the simple blend (crosses) and polymer matrix (dots) at the same frequency and field range are also shown.

mercial dynamic mechanical analyzer (TA Instruments, DMA2980). The electric field induced strain measurement was conducted by a cantilever dilatometer. ${ }^{24}$

Figure 2 presents the dielectric constant and dielectric loss as a function of frequency at room temperature for the nanocomposite with $10 \mathrm{wt} \%$ decorated o-CuPc. The nanocomposite exhibits a large dielectric constant over a relatively broad frequency range. At a frequency of $20 \mathrm{~Hz}$, the dielectric constant of the nanocomposite is 4186 and even though the volume content of net o-CuPc is much lower $(\sim 3.5 \mathrm{vol} \%)$, its dielectric constant is 167 times higher than that of the simple blend of PU with 16 vol \% o-CuPc (the dielectric constant is 25) and 523 times higher than that of the polymer matrix (the dielectric constant is 8). In comparison, the dielectric constant of the simple blend is only three times higher than that of the polymer matrix. Colossal dielectric constants have been reported recently for different classes of materials. Ceramic systems with high dielectric response has been observed in a perovskite-related oxide. ${ }^{25}$ In addition, giant dielectric constant values were observed and correlated to the formation of gain boundary barrier layers and interfacial polarization and percolation effects. ${ }^{16,26}$ Our nanocomposite films have a complex microstructure and mobile charge carriers in the decorated o-CuPc which could result in an enhanced dielectric response caused by the Maxwell-Wagner space charge as reflected by the strong frequency dispersion of the dielectric responses. ${ }^{27,28}$ What is unique is that, although the self-assembled nanocomposite exhibits such a high dielectric response, the net volume fraction of o-CuPc is about 3.5\% in the nanocomposite. Even considering the PAA-decorated o-CuPc as a whole, the vol- 


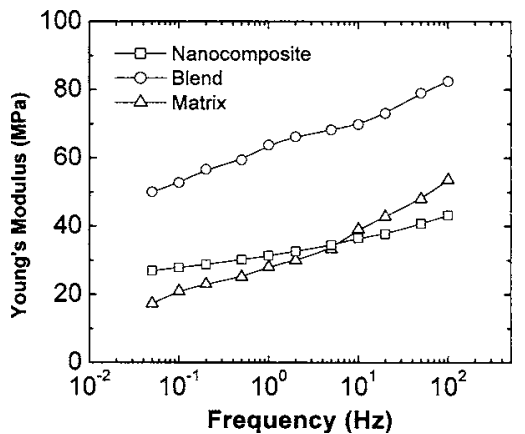

FIG. 3. The elastic modulus measured at room temperature for the nanocomposite, PU matrix, and simple blend.

ume fraction is still below 7.5\%. Such a high dielectric constant could be a result of the nanostructure of the composites in which the high dielectric fillers are concentrated in a low volume fraction continuous phase such as the soft segment in PU. This effect has been made use of to generate conduction percolation at very low conductive polymer volume percentage (one order of magnitude reduction in the percolation threshold) in a self-assembled polymer nanocomposites. ${ }^{23} \mathrm{~A}$ similar phenomenon was also observed in several multiphase composites. $^{16}$

The high dielectric constant nanocomposite also leads to high electromechanical response. The field-induced strain was measured at different applied fields at room temperature and under an ac field of $1 \mathrm{~Hz}$. Presented in Fig. 2(b) is the strain amplitude measured versus the applied-field amplitude for the self-assembled nanocomposite. A strain of $8.3 \%$ can be induced under a field of $11.5 \mathrm{~V} / \mu \mathrm{m}$. The elastic modulus of the nanocomposite was also measured and is presented in Fig. 3. There is no marked change in the elastic modulus in the nanocomposite from that of the PU, presumably due to the low volume fraction of the o-CuPc in the self-assembled nanocomposite. Combining the strain and elastic modulus data yields the elastic energy density of $0.11 \mathrm{~J} / \mathrm{cm}^{3}$ for the nanocomposite under a field of $11.5 \mathrm{~V} / \mu \mathrm{m}$. For comparison, the induced strain from the simple blend as well as polymer matrix is also presented. Under the same electric field, only $0.6 \%$ strain can be induced in the simple blend with $16 \mathrm{vol} \%$ $\mathrm{o}-\mathrm{CuPc}$, which is 1.7 times of the strain of the polymer matrix, $0.36 \%$. The elastic modulus of the polymer matrix is 28 $\mathrm{MPa}$ and the one for the simple blend is $64 \mathrm{MPa}$, which yields the $1.8 \times 10^{-4} \mathrm{~J} / \mathrm{cm}^{3}$ elastic energy density for the polymer matrix and $1.15 \times 10^{-3} \mathrm{~J} / \mathrm{cm}^{3}$ for the simple blend under a field of $11.5 \mathrm{~V} / \mu \mathrm{m}$. The results indicate that the self-assembled nanocomposite can generate a much higher strain as well as elastic energy density compared with the simple blends. Such enhancement can be partly attributed to exchange coupling that is very effective when the size of second phase particles approaches nanometer scale and there are large electric field fluctuations in polymeric matrix due to the large contrast of constituent dielectric constants. ${ }^{29}$
In summary, a route for producing polymeric nanocomposites is demonstrated in which decorated high dielectric constant oligomer-CuPc nanofillers are forced into threedimensional networks by the self-assembly of colloidal particles and segmented copolymers. We also demonstrate the utility of such nanocomposite in generating high dielectric constant and electromechanical response.

This work was supported by NIH under Grant No. 8R01EB002078-04.

${ }^{1}$ Handbook of Low and High Dielectric Constant Materials and Their Applications, edited by H. S. Nalwa (Academic, London, 1999).

${ }^{2}$ Electroactive Polymer (EAP) Actuators as Artificial Muscles-Reality, Potential, and Challenges, 2nd ed., edited by Y. Bar-Cohen (SPIE, Bellingham, WA, 2004).

${ }^{3}$ S. Ashley, Sci. Am. 289, 52 (2003).

${ }^{4}$ Q. M. Zhang, V. Bharti, and X. Zhao, Science 280, 2101 (1998).

${ }^{5}$ R. Pelrine, R. Kornbluh, Q. Pei, and J. Joseph, Science 287, 836 (2000).

${ }^{6}$ H. Engelkamp, S. Middelbeek, and R. J. M. Nolte, Science 284, 785 (1999).

${ }^{7}$ A. K. Boal, F. Ilhan, J. E. DeRouchey, T. Thurn-Albrecht, T. P. Russell, and V. M. Rotello, Nature (London) 404, 746 (2000).

${ }^{8}$ B.-H. Sohn, J. M. Choi, S. I. Yoo, S. H. Yun, W. C. Zin, J. C. Jung, M. Kanehard, T. Hirata, and T. Teranishi, J. Am. Chem. Soc. 125, 6368 (2003).

${ }^{9}$ Q. M. Zhang, H. Li, M. Poh, H. Xu, Z. Y. Cheng, F. Xia, and C. Huang, Nature (London) 419, 284 (2002).

${ }^{10}$ H. Pohl and M. Pollak, J. Chem. Phys. 66, 4031 (1977).

${ }^{11}$ J. Wang, Q. D. Shen, H. M. Bao, C. Yang, and Q. M. Zhang, Macromolecules 38, 2247 (2005).

${ }^{12}$ T. J. Marks, Science 227, 881 (1985); Angew. Chem. 102, 886 (1990).

${ }^{13}$ M. Kimura, K. Wada, K. Ohta, K. Hanabusa, H. Shirai, and N. Kobayashi, Macromolecules 34, 4706 (2001).

${ }^{14}$ F. Garnier, A. Yassar, R. Hajlaoui, G. Horowitz, F. Deloffre, B. Servet, S. Ries, and P. Alnot, J. Am. Chem. Soc. 115, 8716 (1993).

${ }^{15}$ C. Huang and Q. M. Zhang, Adv. Mater. (Weinheim, Germany) 17, 1153 (2005).

${ }^{16}$ C. Huang, Q. M. Zhang, G. deBotton, and K. Bhattacharya, Appl. Phys. Lett. 84, 4391 (2004).

${ }^{17}$ Q. Li and J. A. Lewis, Adv. Mater. (Weinheim, Germany) 19, 1639 (2003).

${ }^{18}$ T. Ngai, G. Zhang, X. Li, D. K. P. Ng, and C. Wu, Langmuir 17, 1381 (2001).

${ }^{19}$ M. Lazzari, and M. A. López-Quintela, Adv. Mater. (Weinheim, Germany) 15, 1583 (2003).

${ }^{20}$ M. Kimura, K. Wada, K. Ohta, K. Hanabusa, H. Shirai, and N. Kobayashi, J. Am. Chem. Soc. 123, 2438 (2001).

${ }^{21}$ M. Kimura, T. Mnto, H. Takimoto, K. Wada, K. Hanabusa, and N. Kobayashi, Langmuir 16, 2078 (2000).

${ }^{22}$ J. Kim, D. Shin, K. Ihn, and C. Nan, Macromol. Chem. Phys. 203, 2454 (2002).

${ }^{23}$ R. Mezzenga, J. Ruokolainen, G. Fredrickson, E. Kramer, D. Moses, A. J. Heeger, and O. Ikkala, Science 299, 1872 (2003).

${ }^{24}$ J. Su, P. Moses, and Q. M. Zhang, Rev. Sci. Instrum. 69, 2480 (1998).

${ }^{25}$ C. Homes, T. Vogt, S. M. Shapiro, S. Wakimoto, and A. Ramire, Science 293, 673 (2001).

${ }^{26}$ Y. Lin, J. Wang, L. Jiang, Yu Chen, and C. W. Nan, Appl. Phys. Lett. 85, 5664 (2004).

${ }^{27}$ A. Turik, G. Radchenko, A. Chernobabov, and A. Khasabov, Ferroelectrics 307, 171 (2004).

${ }^{28}$ P. Lunkenheimer, V. Bobnar, A. Pronin, A. Ritus, A. Volkov, and A. Loidl, Phys. Rev. B 66, 052105 (2002).

${ }^{29}$ J. Y. Li, Phys. Rev. Lett. 90, 217601 (2003). 